\title{
UN CONTROVERTIDO RETRATO DE CARLOS II EN ROMA*
}

El presente artículo estudia un retrato de Carlos II prácticamente desconocido. Su interés se centra, aparte de su calidad pictórica y estética, sobre todo en los sugerentes interrogantes históricos, iconográficos e interpretativos que plantea.

Palabras clave: Pintura; Siglo XVII; Retratos regios; Leopoldo I; Iglesia de Santa Bibiana; Carlos II.

This article concerns a pratically unknown portrait of Charles II of Spain. Aside from its pictorial and esthetic quality, this work is of particular interest because of the suggestive historical, iconographical and interpretative questions to which it gives rise.

Key words: Painting; $17^{\text {th }}$ century; Royal portraits; Leopold I; Church of Saint Bibiana; Charles II of Spain.

Como en tantas ocasiones ocurre en las más variadas facetas de la vida, solemos aceptar aquello que nos viene dado, sin ni siquiera pararnos a reflexionar sobre ello; y sin embargo, en muchas ocasiones, detrás de la aparente simplicidad se ocultan interesantes cuestiones sobre las que merece la pena detenerse. Por ello quería apuntar aquí brevemente la existencia de un controvertido y enigmático retrato de Carlos II (figs. 1 y 2) que, si bien no absolutamente inédito, nunca se había afrontado planteando todos los interrogantes, problemas e hipótesis que sugiere, lo que, precisamente, lo convierte en particularmente interesante.

Dicha efigie se encuentra incluida en forma de medallón, en un lienzo que se conserva en la pequeña y encantadora iglesia romana de Santa Bibiana ${ }^{1}$, condenada al más absoluto olvido junto a los muros de la estación central de Termini ${ }^{2}$. Allí, en la primera capilla del lado de la Epístola $^{3}$, se ven tres lienzos, dos de los cuales, los laterales, contienen sendos medallones con

* Quisiera manifestar mi agradecimiento tanto al párroco de la iglesia de Santa Bibiana como a Domenico Cassano por haberme facilitado el acceso y libre estudio de los lienzos, así como por el permiso para la obtención de las fotografías cuya realización debo a mi compañero en la Academia y gran fotógrafo, Fernando Maquieira. Agradezco muy especialmente al Prefecto del Archivo de Santa Maria Maggiore, Monseñor Michal Jagosz y a su ayudante, el trato y las facilidades dispensadas en el archivo de la Basílica, donde se ha desarrollado gran parte de la investigación para este artículo.

1 Tormo, Elías, Monumentos de españoles en Roma, y de portugueses e hispano-americanos, Madrid, 1942, tomo II, pp. 115-116.

2 Como tantas veces ocurre en las iglesias de Roma, contiene en su interior tesoros artísticos tales como la escultura de la Santa, realizada por Bernini, o los frescos de Pietro da Cortona y Agostino Ciampelli. Fue restaurada hace pocos años; véase Tiberia, Vitaliano, Gian Lorenzo Bernini, Pietro da Cortona, Agostino Ciampelli in Santa Bibiana a Roma: i restauri, Roma, 2000.

${ }^{3}$ Intitulada a la Virgen y a San Flaviano, padre de Santa Bibiana. 


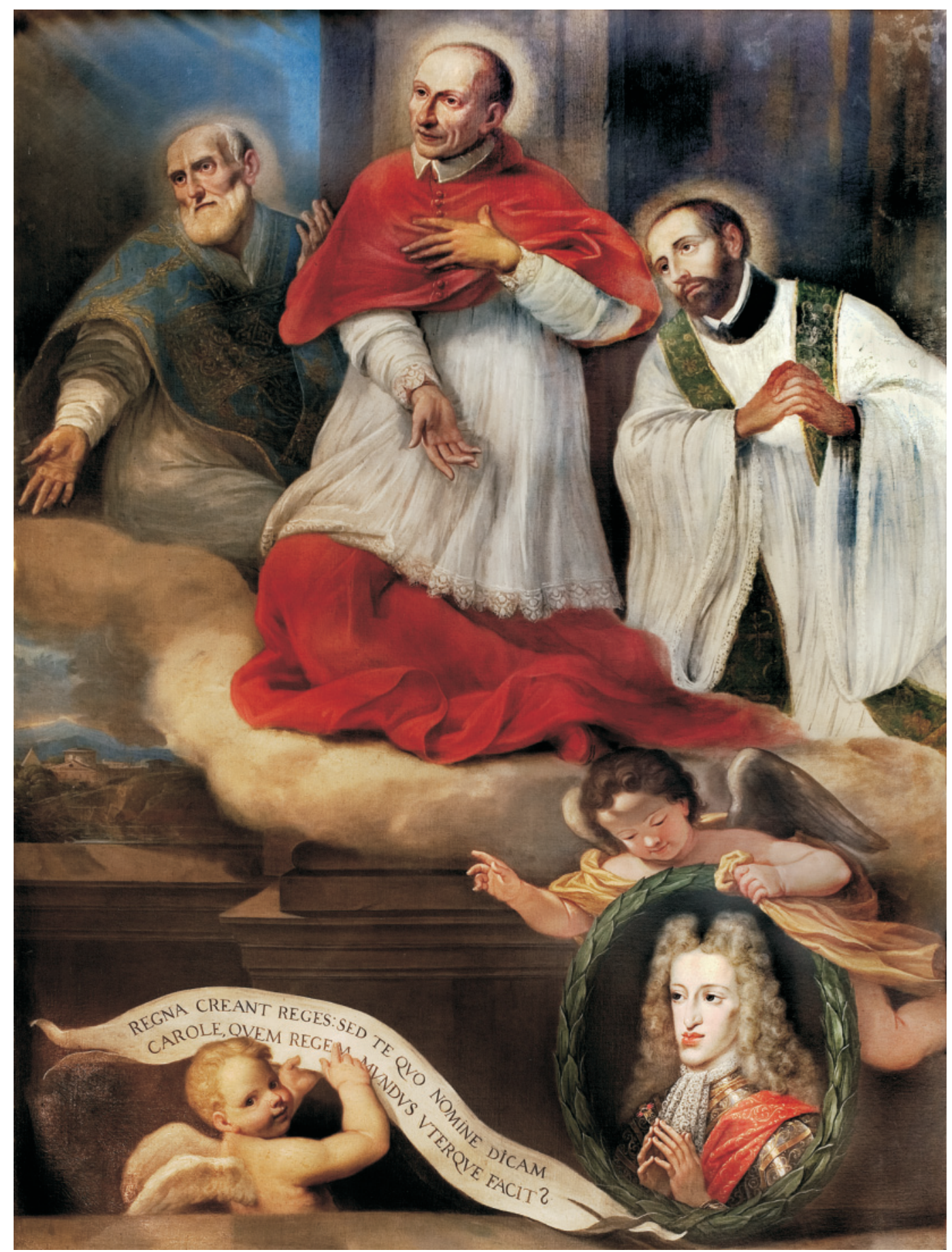

Fig. 1. Anónimo italiano, ¿Giacinto Calandrucci?, San Carlos Borromeo, San Felipe Neri y San Francisco Javier con el retrato de Carlos II, Roma, Iglesia de Santa Bibiana. 
retratos. Dicha capilla fue mandada construir, decorar, adornar y proveer de las correspondientes reliquias, así como de todos los accesorios necesarios para la celebración de misas, en 1700 por el presbítero español, canónigo de la capilla paulina de Santa Maria Maggiore, Francisco de San Juan y Bernedo ${ }^{4}$, estableciendo misas y oraciones pidiendo por la paz y exaltación de la Iglesia, por la salud espiritual y corporal del rey católico así como por la prosperidad de su Monarquía 5 . Todo cual lo explica brevemente también una lápida externa situada a la derecha de la capilla que lleva la fecha de $1702^{6}$, año de la institución del iuspatronato y donación de la Capilla a la familia Petroni, como detallaremos más adelante. Como veremos, todo esto resulta de fundamental importancia a la hora de intentar esbozar las variadas hipótesis plausibles sobre la identidad del personaje, así como la posible explicación del programa decorativo.

Como decimos, en los laterales de la pequeña capilla, hay dos cuadros interesantes que muestran sendos medallones con retratos. El de la izquierda representa a San Juan Bautista, San Juan Evangelista y Santiago el Mayor con el retrato del emperador Leopoldo I (fig. 3), cuya identificación, en este caso, no presenta ningún problema debido a la inscripción que porta un angelote en la parte inferior (Hic Leopoldus Hic Est Pietate Augustus: His Terrea, Illa Astris Intulit Imperium, "Este es Leopoldo, excelso en la fe y en las armas: con éstas ha dirigido el imperio sobre la tierra, con aquella lo ha elevado hasta el cielo"). Ahora bien, mucho más problemático y, quizá por ello, también más interesante, es el cuadro gemelo ${ }^{7}$ que hay enfrente. Se representa en éste a San Carlos Borromeo, San Felipe Neri y San Francisco Javier ${ }^{8}$, que amparan el medalón con el retrato de otro personaje importante. En la parte inferior, otro angelote sostiene una especie de filacteria con la inscripción: Regna Creant reges sed te quo Nomine Dicam Carole Quem Regem Mundus Uterque Facit, ("Los reinos crean reyes, pero a ti, Carlos, con qué nombre te llamaré a quien ambos mundos hacen rey"). Es aquí donde comienzan las incógnitas. Para Tormo no hay duda de que se trata del segundo hijo varón del emperador, el archiduque Carlos de Austria, pretendiente a la Corona de España, siendo realizados ambos retratos en vida aún del emperador Leopoldo ${ }^{9}$ (que efectivamente muere en 1705, y, como hemos visto, la capilla se fecha en 1700). Ahora bien, el mismo Tormo se hace eco del tremendo parecido con Carlos II, aludiendo a la proximidad de los rasgos fisiognómicos de los Austrias y reconociendo el uso de la peluca "a la francesa" 10 que sabemos utilizó Carlos II después de perder mucho pelo tras una

\footnotetext{
${ }^{4}$ Natural de Puente la Reina, en Navarra. La licencia para la capilla fue otorgada por el Capítulo de Santa María Maggiore el 23 de octubre de 1700. Archivio della Basilica Papale di Santa Maria Maggiore (en adelante ABPSMM), Chiesa di Santa Bibiana. Cappellanie e obblighi di messe, stanza 1, armadio 32, ripiano 3, busta/fasc 827/05. Capellania in S. Bibiana del S D Francesco di S Gio e Bernedo da puente della Reina, f. $1 \mathrm{r}$ ( $\sin$ foliar, la numeración es mia).

5 ABPSMM, Chiesa di Santa Bibiana. Cappellanie e obblighi di messe, stanza 1, armadio 32, ripiano 3, busta/fasc 827\05. Copia della tabella che si conserva nella sagrestia di S. Bibiana della fondazne della Capla eretta dal gm D. Franco di S. Gio e Bernedo sotto l'invocazione della SS Vergne e S. Flaviano M in d Chiesa..., f. 2r (sin foliar, la numeración es mia). El documento lleva la fecha de 1707, y como se indica en el mismo, se realiza ésta copia con el fin de que quede memoria, pues el original "si va porcando, e consumando ch'apena si può leggere", tanto es así que, efectivamente, no se conserva.

${ }^{6}$ Forcella, Vincenzo, Inscrizioni delle Chiese e d'altri edificii di Roma dal secolo XI fino ai giorni nostri, volume XI, Roma, 1877, p. 122. Existe un documento contemporáneo en el que se transcribe ya la inscripción de la lápida. ABPSMM, Chiesa di Santa Bibiana. Cappellanie e obblighi di messe, stanza 1, armadio 32, ripiano 3, busta/fasc $827 \backslash 05$, folio suelto.

7 Ambos miden $237 \times 172 \mathrm{~cm}$.

8 Vasco Rocca, Sandra, Santa Bibiana, Roma, 1983, p. 84. Tormo, Elías, Monumentos de españoles..., op. cit., p. 115. San Francesco Saverio en italiano.

9 Tormo, Elías, Monumentos de españoles..., op. cit., p. 115. Equivoca el grado de parentesco, señalando al archiduque Carlos como hermano de Leopoldo I.

10 Ibidem.
} 


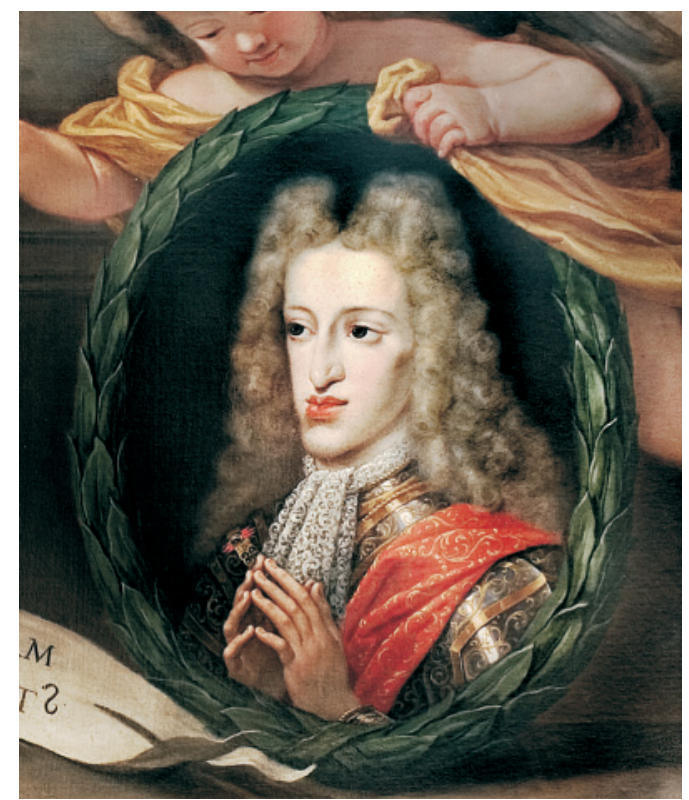

Fig. 2. Particular del retrato de Carlos II.

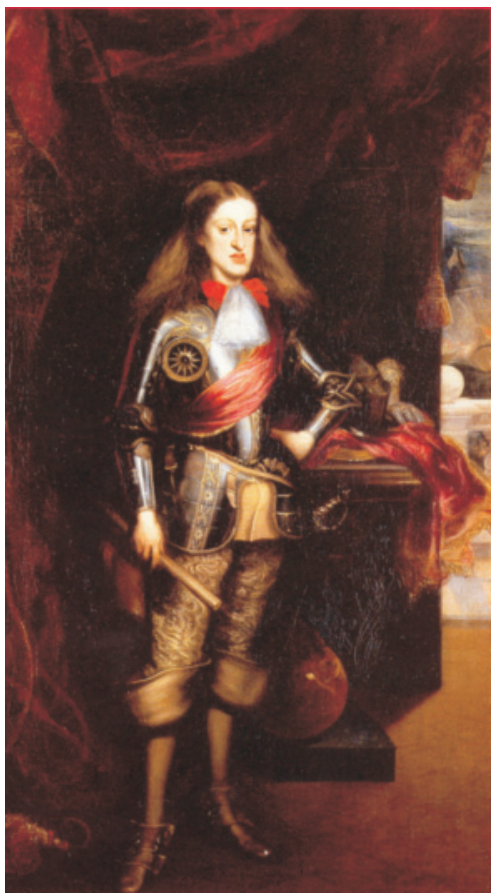

Fig. 4. Juan Carreño de Miranda, Carlos II con armadura, Toledo, Museo del Greco@.

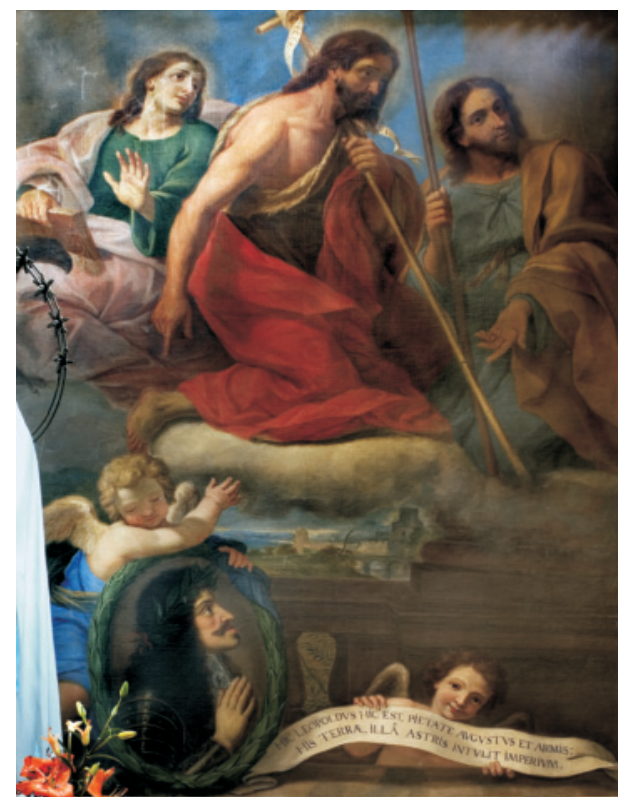

Fig. 3. Anónimo italiano, Giacinto Calandrucci?, San Juan Bautista, San Juan Evangelista y Santiago el Mayor con el retrato de Leopoldo I, Roma, Iglesia de Santa Bibiana.

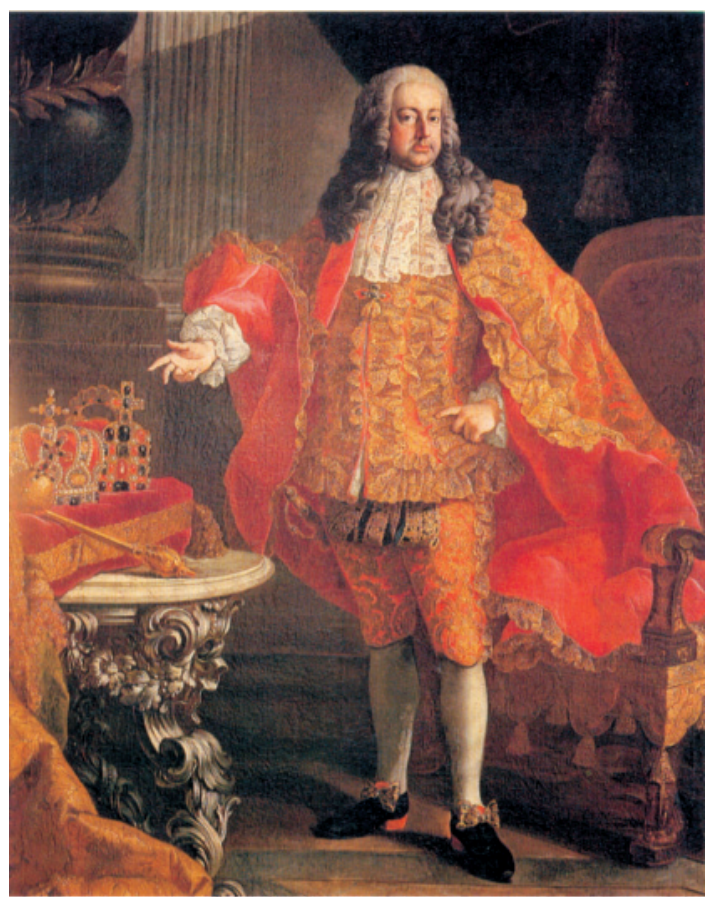

Fig. 5. Martín von Meytens, Carlos VI de Austria, Florencia, Galeria degli Uffizi(C). 
de sus numerosas enfermedades ${ }^{11}$. Por su parte, Vasco Rocca lo identifica, también sin duda, con Carlos II, por lo que según ella la datación de los cuadros no podría superar la fecha de la muerte del soberano español, aduciendo también que las inscripciones que acompañan a ambos retratos se refieren claramente a personas vivas ${ }^{12}$, lo cual, de paso, podría ser más que discutible. Vayamos por partes.

Ante esto se nos plantean varios interrogantes y dificultades que nos ofrecen otras tantas hipótesis, sin que pretendan éstas ser nada más que eso. Sin embargo he de decir que, desde que vi el cuadro en Roma y a pesar de la identificación que hace Tormo, nunca llegué a convencerme de que el que allí aparece efigiado sea afectivamente aquel Carlos, sino nuestro Carlos. Convicción que recibe cabal confirmación a través de un documento que hemos localizado, fechado en abril de 1727, en el que se describe la iglesia, mencionándose los cuadros e identificando ya ambos personajes como el Emperador Leopoldo y Carlos II, así como los santos que les acompañan ${ }^{13}$.

A la evidencia documental se une la empírica, pues resulta obvio a simple vista que en realidad el lienzo no figura el rostro del archiduque, sino el del Carlos II, muy similar a como le vemos en el retrato del Museo del Greco en Toledo (fig. 4). Como hemos visto, incluso Tormo, a pesar de la identificación, reconocía el tremendo parecido con Carlos II. De hecho en los retratos que conocemos del archiduque ${ }^{14}$ (fig. 5), su rostro no es, ni de lejos, tan idéntico al de Carlos II, por mucho que se quiera atribuir esta sorprendente semejanza al "aire de familia" como hace Tormo.

Ahora bien, a pesar de la errónea identificación que hace Tormo, su aguda intuición no creemos que estuviese del todo desencaminada. Es evidente que ambos lienzos están colocados con una intención no sólo decorativa sino formando parte de un programa de hondo calado significante en unas fechas decisivas para el futuro y posterior evolución de la Monarquía Católica. Parece claro que lo que llevó a Tormo a identificar al personaje con el Archiduque Carlos es precisamente la ambigua inscripción que aparece en el retrato, pues como el mismo dice "Por la letra induzco que éste no es aún emperador Carlos VI, sino aun rey"15.

Efectivamente, a pesar de que la identificación del personaje parece haber quedado demostrada, siguen surgiendo dificultades al respecto pues existen varios aspectos problemáticos que no terminan de encajar entre sí. Sobre todo en lo que se refiere a la datación de la construcción y decoración de la capilla, la inscripción de la lápida, y, muy especialmente, a la misma del cuadro, así como lo que ambos lienzos suponen desde el punto de vista programático. Todo lo cual se nos presenta estrecha e indisolublemente relacionado entre sí, veamos porqué. En primer lugar se encuentra todo lo referente a la construcción y datación de la capilla, de fundamental importancia a la hora de plantear las posibles explicaciones e implicaciones del programa decorativo. Como hemos mencionado más arriba, la lápida que se encuentra fuera de la capilla explica quién fue el comitente de la obra, ofreciéndonos así mismo la fecha de 1702 y el motivo de la construcción y decoración por la paz y exaltación de la Santa Madre Iglesia así como por la salud del Rey Cató-

11 El embajador imperial, conde Wenzel de Lobkowitz escribía al emperador: "el Rey ha perdido mucho pelo después de su enfermedad y dice que para tapar la calva se pondrá una peluca, pero sin rizos ni polvos, por no parecerse al Francés, a quien odia por tantas razones"; Maura, Duque de, Vida y reinado de Carlos II, Madrid, 1990, p. 413 (primera edición, 1942).

12 VAsco Rocca, Sandra, Santa Bibiana, op. cit., pp. 84-85.

13 ABPSMM, Chiesa di Santa Bibiana. Descrizione chiesa 1705-1866, stanza 1, armadio 32, ripinao 3, busta/fasc 827\07. Iventario e stato della Chiesa di Santa Bibiana unita alla Basilica di Santa Maria Maggiore, ff. 4r-v (sin foliar, la numeración es mía). El documento lleva la fecha de 20 o 30 (imposible de distinguir) de abril de 1727.

${ }^{14}$ Sobre el personaje y su descripción física, véase la reciente monografía de LEón, Virginia, Carlos VI. El emperador que no pudo ser rey de España, Madrid, 2003.

15 Tormo, Elías, Monumentos de españoles..., op. cit., p. 115. 
lico. En principio, esto parecería entrar en contradicción con que el retratado sea Carlos II, pues parece un tanto absurdo rogar por la salud del rey cuando este estaba ya muerto. Sin embargo, como ya se ha apuntado más arriba y se desprende de la documentación, esta fecha no corresponde a la de fundación, construcción y decoración de la capilla, sino a la institución del iuspatronato y donación de la misma al Conde Francisco María Petroni (por el que el comitente profesaba un gran afecto) y, a través de él, a todos los herederos de la familia Petroni. Así se refleja de modo claro en el documento ya citado de 1702, en el que, ante notario, Francisco de San Juan y Bernedo "dichiarando di haver fabricata a sue prorprie spese una capella nella Chiesa di S ta $^{\text {B }}$ Bibiana [...] con licenza dell'Illust ${ }^{\text {mo }}$ Capitolo della Sacros. Patriarcale Basílica di Sta Maria Maggiore, come dice apparire per suo decreto fatto li 23 ottobre 1700 [...] con haverla arrichita con molte Reliquie de SS Martiri [...], ornata di piture, provista di candelieri con una croce, tovaglie per il altare, fiori con suoi vasi [...] E trovandosi esso Sign ${ }^{r e}$ D. Franceso in età molto avanzata, e non haver Parenti pero per l'afetto, et amore che porta all'Illust ${ }^{\text {mo }}$ Conte Francesco Maria Petroni [...] habbia donato per donazione irrevocabile che si dice fare inter vivos alli medessi la sudetta Capella e ius honorifico di essa con tutti li suoi arredi ${ }^{16}$. En otra parte del documento aún se es más explícito pues Francisco de San Juan y Bernedo, hablando ya en primera persona, indica que "trovandomi molto innnazi nell'eta, e non haver Parenti [...] dono per donazione irrevocabile inter vivos la sud ${ }^{a}$ Capella a sia ius honorifico con tutte le pitture [...] Reliquie, et altro in essa essistente tanto sino ad hora fatto, quanto che potessi fare in avenire per servizio, et ornamento della medemma Capella all'Illust ${ }^{\text {mo }}$ Sign $^{\text {re }}$ Conte Francesco Maria Petroni" ${ }^{17}$. Como se puede comprobar en la documentación, se habla siempre en pasado, por lo que resulta evidente que en esa fecha de 1702 la capilla estaba ya construida, decorada y dotada de todo lo necesario. Es asimismo claro que debió encargarse y empezar a construirse aún en vida del rey, apenas se obtuvo la licencia para la misma (y aún antes) a finales de octubre de 1700. Precisamente por ello, tendría pleno sentido pedir por su salud en tan amargo trance, pues por aquellas fechas, Carlos II se encontraba ya gravemente enfermo y a nadie se le escapaba que eran sus últimos momentos en este mundo (moriría de hecho el 1 de noviembre). La noticia de la muerte del rey llegó pronto a Roma ${ }^{18} \mathrm{y}$, más aún, a la Basílica de Santa María Maggiore precisamente de patrocinio Hispano, donde consta documentalmente que se organizaron unos fastuosos funerales regios el 22 de enero de $1701^{19}$. Así pues, resulta evidente que en el momento de colocación de la lápida era perfectamente conocida la muerte del rey, y no entra dentro de lo razonable pensar que precisamente el comitente de la capilla desconociese la noticia, máxime cuando además era canónigo de Santa Maria Maggiore donde, como acabamos de ver, se habían celebrado las solemnes exequias fúnebres por Carlos II. Por tanto, por mucho que la lápida lleve la fecha de 1702, recuerda y hace referencia al momento fundacional de la capilla que, como llevamos dicho, corresponde a 1700.

Cuestión aparte y sin duda la que reviste mayor interés, convirtiéndose de hecho en la clave de todo el asunto, es la de porqué, a pesar de que el rey había muerto y Felipe V había sido ya proclamado rey de España siguiendo las cláusulas testamentarias, el representado sigue siendo Carlos II y no se actualizó la imagen del rey. Quizá la respuesta más sencilla y evidente corres-

16 ABPSMM, Chiesa di Santa Bibiana. Cappellanie e obblighi di messe, stanza 1, armadio 32, ripiano 3, busta/fasc 827\05. Capellania in S. Bibiana del S D Francesco di S Gio e Bernedo da puente della Reina, f. 1r (sin foliar, la numeración es mia).

17 Ibid., f. $1 \mathrm{v}$.

18 Concretamente el 21 de noviembre de 1700; véase VALEsio, Francesco, Diario di Roma. Libro primo e secondo, volume I: 1700-1701, edizione di Milano, 1977, p. 124.

19 ABPSMM, Relazione dell'esequie reali fatte Nella Sacro-Santa Basilica di S. Maria Maggiore In Roma à dì 22 Gennaro 1701 Per il già Rè delle Spagne Carlo II Di sempre Gloriosa Memoria. In Roma, MDCCI, stanza 1, armadio 23, ripiano 2, busta/fasc 460\02. 
ponda a una cuestión práctica, pues toda la capilla, incluidos obviamente los cuadros, fueron encargados y acaso estuvieron listos antes de conocerse la noticia de la muerte del rey, por lo que tal vez se decidió dejar todo tal cual, debido a las complicaciones adicionales y el gasto suplementario que hubiese supuesto una renovación decorativa al respecto, pues no olvidemos que, tanto la arquitectura como la decoración y complementos necesarios de la capilla, corrieron exclusivamente a cargo de Francisco de San Juan y Bernedo.

Ahora bien, detrás de todo esto, da la impresión de que existe algo más, de que lo que vemos no responde a la simple praxis o casualidad, sino que está intencionadamente buscado con una finalidad trascendente, conformando un auténtico programa cargado de intención. Es decir, parece esconderse un verdadero posicionamiento político del comitente que, precisamente, podría ofrecernos un sentido y explicación convincente del programa.

En este sentido, la cuestión más espinosa es la ambigua inscripción que aparece en el cuadro, que es la que, en realidad, no deja nada clara la identidad del retratado, pudiéndose plantear varias hipótesis o razonamientos al respecto. Es justamente dicha inscripción, la que nos ofrece algunas de las pistas para una posible interpretación del sentido y significado del programa decorativo que ambos lienzos conforman. Pues es evidente que, a pesar de todo lo que llevamos dicho, siguen existiendo cuestiones que continúan sin cuadrar. Recordémosla: "Los reinos crean reyes, pero a ti, Carlos, con qué nombre te llamaré, a quien ambos mundos hacen rey". Si se trata de Carlos II, no se entiende la pregunta retórica de "con que nombre te llamaré", es decir ¿porqué no se incluye el nombre completo de Carlos II y además se habla en futuro? Si es Carlos II el representado, no había necesidad alguna de pensar en un nombre con el que habría de ser llamado en el futuro.

Por otra parte está el hecho de que diga "a quien ambos mundos hacen rey", que también se podría traducir por "a quién uno y otro mundo hacen rey". La cuestión es ia que mundos se refiere? Si se trata del viejo mundo y América, es decir, la titulación habitual como Hispaniarum et indiarum rex, tendría sentido que se tratase de Carlos II, pero ¿y si en realidad se refiere a este y el otro mundo, es decir, el más allá, de los que también habla la inscripción del retrato de Leopoldo? (tierra y cielo). Da la impresión pues de que se trata o bien de una persona muerta a quién se dedica el cuadro, en cuyo caso no tiene entonces sentido la primera parte de la inscripción; o de que se alude a otra persona que no es Carlos II.

Dichas incógnitas parecerían dar la razón a Tormo en su identificación con el archiduque Carlos a pesar de las evidencias mostradas. En primer lugar, en la fecha aproximada de realización de los lienzos (esto es, a finales de 1700) no parece tener mucho sentido la inclusión de un retrato del emperador con otro de Carlos II haciendo pareja, cuando ya Carlos II había nombrado heredero al nieto del rey de Francia, en detrimento de los intereses del emperador, que luchaba por los derechos de su hijo a la Corona de España. Precisamente además, como hemos visto, se recibió la licencia para la capilla poco después de que Carlos II dictase su último testamento a principios de octubre, en el que dejaba todas las posesiones españolas al Duque de Anjou ${ }^{20}$. Tal vez fuese precisamente este hecho, el revulsivo que impulsó a encargar la capilla como medio de mostrar sutilmente la posición política del comitente.

Por otra parte, aunque en estas fechas todavía no había comenzado de modo efectivo la guerra de sucesión, el archiduque Carlos, con el emperador Leopoldo a la cabeza, se había perfilado ya como el oponente claro a Felipe $\mathrm{V}^{21}$. En este contexto tendría entonces pleno sentido la ins-

20 Domínguez Ortiz, Antonio, "Introducción al testamento de Carlos II" en Testamento de Carlos II, Madrid, 1980, pp. I-LVII.

${ }^{21}$ La síntesis más completa sobre este tumultuoso momento inmediatamente anterior y posterior a la muerte de Carlos II, así como todo el desarrollo posterior de la Guerra de Sucesión se encuentra en La transición del siglo XVII al XVIII. Entre la decadencia y la reconstrucción, tomo XXVIII de la Historia de España Menéndez Pidal, Madrid, 1993, pp. $145-155$ y 303-503 
cripción del retrato: se aludiría a la evidente victoria del archiduque Carlos por voluntad divina, preguntándose por el nombre con que será llamado una vez ésta haya sido conseguida y se haya convertido en rey de las Españas, incluidas, obviamente, las Indias (ambos mundos).

Es decir, a pesar de que parece haber quedado demostrado que el representado es sin ningún lugar a dudas el rey Carlos II, se produce un doble juego al que se presta muy bien la coincidencia entre el nombre del Rey y el del pretendiente. Es esto precisamente lo que permite una ingeniosa combinación entre texto e imagen, pues si por un lado se representa a Carlos II, la vaga, confusa y ambigua inscripción del lienzo deja entrever el posicionamiento o filiación política del comitente, hacia la continuidad de la Casa de Austria en el trono hispano concretada en la figura del Archiduque Carlos. Da la impresión pues, de que todo el programa se convierte en un sutil alegato a favor del pretendiente austriaco, presentándole como legítimo y natural heredero en el que confluyen las dos ramas de la Casa de Austria, de ahí la presencia del emperador Leopoldo I y del rey Carlos II.

Respecto al posible autor del cuadro es bien poco lo que conocemos, pues ambos lienzos, que fueron restaurados en los años ochenta, no aparecen mencionados en las guías de la época ${ }^{22}$, lo cual parecería indicar el escaso renombre del pintor ${ }^{23}$. La similitud estilística y compositiva entre ambos nos habla de un mismo autor, aunque el retrato de Carlos II, resulta de mejor calidad que el del emperador ${ }^{24}$. Se ha apuntado la posibilidad de que respondan al estilo del pintor palermitano Giacinto Calandrucci ${ }^{25}$, que, después de una inicial formación en Palermo, se trasladó a Roma donde estudió primero con Pietro del Pò, pasando poco después al taller de Carlo Maratti del que se convirtió en uno de sus más fieles discípulos y colaboradores ${ }^{26}$. A este respecto, debemos hacer notar que en la Colegiata de Canino (Viterbo) se conserva una Virgen con el niño, San Felipe Neri y San Francisco, obra de Giacinto Calandrucci ${ }^{27}$, que se acercó mucho estilísticamente al lienzo de la iglesia de Santa Bibiana. Encontramos asimismo serias concomitancias desde el punto de vista compositivo e iconográfico, sobre todo en lo que a la figura de San Felipe Neri se refiere, así como en el significativo detalle de la presencia del retrato de un personaje que une sus manos en oración, a modo de donante, situado en la esquina inferior izquierda. Del mismo modo, en la iglesia parroquial de Camerano (provincia de Ancona), existe una Aparición de la Virgen a San Felipe Neri, de ignoto pintor, aunque asimilado a la labor de Calandrucci, en donde la figura del santo se asemeja mucho iconográfica y estilísticamente al de la iglesia de Santa Bibiana.

Álvaro Pascual Chenel Real Academia de España en Roma

\footnotetext{
${ }^{22}$ Aunque curiosamente si en algún documento casi contemporáneo, como hemos visto.

23 VAsco Rocca, Sandra, Santa Bibiana, op. cit., p. 84, nota 83.

24 Ibid., p. 85.

${ }^{25}$ Ibidem.

${ }^{26}$ SESTIERI, Giancarlo, Repertorio della pittura romana della fine dell seicento e del settencento, vol. Primo, Torino, 1994, pp. 40-42.

27 Graf, Dieter, Die Handzeichmungen von Giacinto Calandrucci, Kunstmuseum Düsseldorf, Düsseldorf, 1986, Text, pp. 110-111; Tafeln, p. 526.
} 